\title{
NK cells in the tumor microenvironment and thioredoxin activity
}

\author{
Theresa L. Whiteside \\ Department of Pathology, Department of Immunology, and Department of Otolaryngology, University of Pittsburgh School of Medicine and UPMC Hillman Cancer Center Pittsburgh, \\ Pittsburgh, Pennsylvania, USA
}

\begin{abstract}
NK cells are responsible for defense against viral infections and cancer. Although activated NK cells are armed to combat tumors, the tumor microenvironment (TME) contains ROS, which suppress NK cell antitumor activity. In this issue of the $J C I$, Yang, Neo, and colleagues explored NK cell resistance to oxidative stress in vitro and in human non-small-cell lung cancer (NSCLC). High surface thiol density and elevated expression of the ROS scavenger thioredoxin (Trx1) protected NK cells from ROS. Trx1 and thiol levels were higher in IL-15- than in IL-2-primed NK cells. Tumor-infiltrating $\mathrm{Trx1}^{+}$NK cells were present in patients with NSCLC with elevated ROS levels in the tumor. Smokers scored higher for the ROS signature, which predicted poor prognosis, compared with nonsmokers. This study explains how activated NK cells survive in the ROS-rich TME and suggests that smokers with lung cancer may benefit from therapies using IL-15-primed NK cells.
\end{abstract}

\section{NK cells and innate immunity}

NK cells are a subset of innate immune cells responsible for defense against infected and malignant cells (1). The first studies of NK cells in the 1980s identified them as lymphocytes that phenotypically were neither $\mathrm{T}$ cells nor B cells and were able to spontaneously, without prior sensitization, eliminate virally infected or malignant cells in vitro and in vivo $(2,3)$. At that time, NK cells were known to express CD56 and CD16, the two markers that even today distinguish NK cell subsets $\left(C D 56^{\text {bright }}\right.$ CD16 ${ }^{\text {dim }}$ from CD56 ${ }^{\text {dim }}$ CD16 $\left.{ }^{\text {bright }}\right)$. The former, smaller subset has an increased capacity to produce inflammatory cytokines, including IFN- $\gamma$ and TNF- $\alpha$, whereas the CD56 ${ }^{\mathrm{dim}} \mathrm{CD} 16^{\text {bright }}$ NK cells mediate cytotoxicity (4).

In the 1990s, molecular mechanisms NK cells use for target killing were defined (5), and what is referred to as the "missing-self" hypothesis was proposed to explain how NK cells recognize "bad" targets and spare "good" cells (6). However, it was the recognition of multiple NK cell surface receptors, including killerIg-like receptors (KIRs) and natural cytotoxicity receptors (NCRs), that provided insights into NK cell signaling and the capability of these cells to engage multiple targets. Within a span of 30 years, NK cells emerged from relative obscurity and assumed a prominent role as major effectors of innate immunity endowed with a rich repertoire of activating and inhibitory receptors (Figure 1). The surface of NK cells is now understood to resemble an intricate puzzle of receptors and ligands that integrate activating and inhibitory signals, ultimately determining NK cell responses in the microenvironment (5).

NK cells in mouse spleen or bone marrow or human PBMCs are known as conventional NK (cNK) cells. Studies of tissue-resident NK (trNK) cells reveal the existence of phenotypic and func-

Related Article: p. 5508

Conflict of interest: The author has declared that no conflict of interest exists.

Copyright: @ 2020, American Society for Clinical Investigation.

Reference information: / Clin Invest. 2020;130(10):5115-5117. https://doi.org/10.1172/JCl141460.

tional heterogeneity that far exceeds the immune surveillance role originally attributed to NK cells. Environmental factors and/or epigenetic modifications of NK cell genes via DNA methylation may shape trNK cell functional diversity (7). Interactions between trNK cells and other cells in tissues result in a context-dependent crosstalk that varies with the tissue site. For example, lymph node-derived versus lung tissue-derived trNK cells or tumorinfiltrating versus nonmalignant tissueresident NK cells show marked phenotypic and functional differences (8).

Tumor-infiltrating NK cells have been of special interest for many years, largely because of their antitumor and antimetastatic potential (9). From the therapeutic viewpoint, the optimization of NK cell migration, activation, survival, and persistence in the tumor microenvironment (TME) is highly desirable. However, the TME is immunosuppressive, and among many mechanisms contributing to immune suppression, oxidative stress is a major contributor (10). ROS are produced by tumor cells but also by other cells in the TME, including activated immune cells. High ROS levels dampen antitumor immune responses in the TME.

\section{NK cells are sensitive to oxidative stress}

In this issue of the JCI, scientists led by Andreas Lundqvist and Kai Wang remind us that NK cells are sensitive to oxidative stress and become profoundly immunosuppressed upon in vitro exposure to $\mathrm{H}_{2} \mathrm{O}_{2}$ (11). The researchers report that NK cells infiltrating human solid tumors were negatively influenced by high ROS levels in the TME. Therefore, the question of how to protect trNK or adoptively transferred NK cells from ROS is relevant to cancer immunotherapies.

The investigators report that IL-15primed NK cells showed an increased ability to resist ROS-mediated immune 
A Circa 1980s

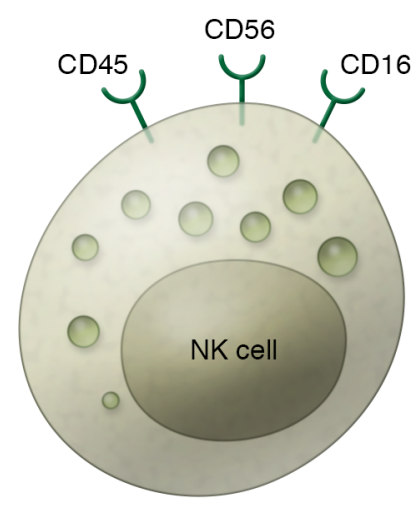

B Today

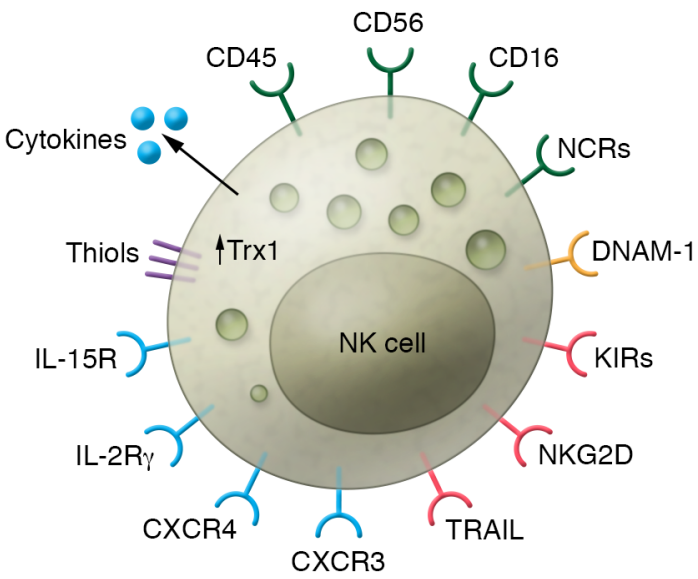

Figure 1. The emergence of NK cells from obscurity to biological prominence. (A) When discovered in the 1980s, NK cells were recognized as CD45+CD56+CD16 ${ }^{+}$effector lymphocytes that did not express the T cell receptor or the B cell receptor. (B) Today, the NK cell surface is decorated by multiple receptors, only some of which are shown above. Sixteen activating proteins (NCRs, green), at least ten different KIRs, and various inhibitory receptors such as NKG2D or TRAIL (red), chemokine receptors (e.g., CXCR3 or CXCR4) and cytokine receptors (including IL-2R and IL-15R, blue), and adhesion molecules (e.g., DNAM-1, yellow) are expressed on NK cells. Further, NK cells produce a variety of cytokines and soluble factors that modulate functions of cells interacting with NK cells. NK cell-associated receptors and ligands are reported with regular frequency, as functionally diverse subsets of trNK cells are discovered and characterized. The study by Yang, Neo, and colleagues (11) reports that Trx1 content increases in activated NK cells and that the levels of thiols (purple) are upregulated on the cell surface, providing a protective screen against ROS.

suppression (11). IL-15 is well known for its ability to activate NK cells. Signaling via STAT5 and engaging the mTOR pathway, IL-15 promotes effector functions (12). It appears that IL-15-primed NK cells have a high content of thioredoxin (Trx), a scavenger for ROS and a known antioxidant (13). In vitro, IL-15, and to a lesser extent IL-2, protected NK cells from functional suppression by $\mathrm{H}_{2} \mathrm{O}_{2}$. The researchers also performed transcriptomic analysis of publicly available sequencing data and compared gene enrichment in IL-15- versus IL-2-primed NK cells obtained from various donors. They observed elevated gene expression of thioredoxins (TXN1 and TXN2) and reduced expression of TXNIP and TXNRD1 genes that code for Trx-interacting proteins, which negatively regulate Trx. A similar differential expression of TXN and TXNIP genes was seen at the mRNA level, and flow cytometry confirmed higher levels of Trx1 in IL-15than in IL-2-primed NK cells. Importantly, $\mathrm{H}_{2} \mathrm{O}_{2}$ induced shuttling of TXNIP from the nucleus to the cytoplasm, and this change in TXNIP localization had a crucial role in the regulation of Trx1 antioxidant activity: it reduced intracellular ROS in IL-15- primed NK cells and promoted restoration of their immune functions.

\section{Thiol density on the NK cell surface}

The researchers from the Lundqvist and Wang laboratories went on to show a causal relationship between Trx1 expression and the density of thiols on the NK cell surface (11). Specifically, Trx1 regulated thiol density on the NK cell surface. Only NK cells with low thiol density accumulated intracellular ROS. Only NK cells with high thiol density were resistant to ROSinduced suppression. These NK cells had high Trx1 levels, upregulated CD56 and CD16 expression, and increased mTOR activity, and they mediated strong antitumor cytotoxicity. In contrast, NK cells with low surface density of thiols accumulated inhibitory TXNIP in the cytoplasm (11). It appears that the surface thiols, reflecting the NK cell content of Trx1, serve as cellular markers of ROS-mediated immune suppression in the TME but also as a potential antioxidant shield or barrier protecting NK cells and neighboring immune cells from oxidative stress. This latter hypothesis was tested using tumor spheroids. NK cells separated by sorting into high thiol- and low thiol-expressing subsets were mixed with cultured autologous $\mathrm{T}$ lymphocytes and compared for the ability to infiltrate spheroids. Tumor-infiltrating lymphocytes (TILs) enriched in thiol-rich NK cells had a greater ability to infiltrate into tumor spheroids than did TILs containing low thiol-expressing NK cells. These data give credence to the notion that thiol-rich $\operatorname{Tr} \times 1^{+}$ NK cells promote TIL infiltration into the tumor by protecting these TILs from oxidative stress in the TME.

Finally, the researchers showed that PX-12, a selective Trx1 inhibitor, prevented IL-15- or IL-2-primed NK cells from killing leukemic K562 target cells. When the authors treated 3D lung adenocarcinoma spheroids with PX-12, IL-15-primed NK cells failed to robustly infiltrate the tumor. To further show that mTOR pathway activation relates to Trx1 and TXNIP in IL-15-primed NK cells, the researchers used a selective mTOR inhibitor (Torin-1). Torin-1 decreased Trx1 levels and surface thiol density but upregulated TXNIP expression (11). In aggregate, these data suggest that activation of the mTOR pathway in IL-15-primed NK cells is critical for regulation of the thioredoxin system and for IL-15-mediated resistance of NK cells to oxidative stress.

\section{Clinical implications}

Lundqvist, Wang, and colleagues translated the results of these in vitro experiments to NK cells infiltrating human non-smallcell lung cancer (NSCLC) by evaluating tumor samples obtained from 29 patients (11). The frequency of CD $56^{+} \mathrm{NK}$ cells was higher in the tumor periphery than within the tumor core. NK cells in the tumor core had higher intracellular ROS than did those in the tumor periphery, an indication that oxidative stress in the TME impacts NK cell activity. Because smoking is associated with increased ROS production, the authors divided the patients with NSCLC into smoker and nonsmoker groups and searched The Cancer Genome Atlas (TCGA) database for associations of prognosis with the ROS gene signature, the NK cell gene signature, and the IL-15 gene signature. They found that smokers had a higher score than nonsmokers for the ROS signature, which predicted a worse prognosis. The NK cell signature predicted 
overall survival and progression-free interval only in the smoker cohort. The IL-15 gene signature was only predictive for progression-free interval in the smoker cohort (11). These results suggest that smokers not only have a high propensity for oxidative stress in the tumor but also that only smokers are likely to benefit from therapies with IL-15-primed NK cells or from therapies with antioxidants aimed at relieving oxidative stress in the TME. This study enlarges our understanding of the fate of activated NK cells in the hostile ROS-rich TME (11). NK cells found in the core of solid tumors, such as NSCLC, accumulated ROS, which impaired their antitumor activities. However, these NK cells were $\operatorname{Trx}^{+}$and resisted the effects of ROS. The role of IL-15 in inducing NK cell resistance to oxidative stress via the thioredoxin system and activated mTOR is critical. The mechanisms used by IL-15-primed NK cells to disarm ROS include shuttling of TXNIP from the nucleus to the cytoplasm and the upregulation of thiols on the NK cell surface. These IL-15-induced, antioxidant-like features of NK cells enable them to deal with high ROS levels and perhaps even provide a protective thiol screen for other lymphocytes in the TME. In NSCLC, tobacco smoking is a risk factor, and smoking is associated with increased ROS production. Based on the TCGA searches, the prognostic value of the IL-15 or NK cell gene signatures in NSCLC appears to be relevant only for smokers. This suggests, albeit only subtly, that antioxidant therapies might be of greater benefit in patients with a history of tobacco use. Taken together, the researchers led by Andreas Lundqvist uncover yet another aspect of NK cell plasticity, whereby priming with IL-15 endows the cells with an increased ability to infiltrate solid tumors, resist ROS-mediated immune suppression, mediate antitumor functions, and perhaps even protect $\mathrm{T}$ cells from ROS in the TME.

\section{Acknowledgments}

TLW is supported in part by award U01DE029759 from the National Institute of Dental and Craniofacial Research (NIDCR), NIH.

Address correspondence to: Theresa L. Whiteside, UPMC Hillman Cancer Center, UPCI Research Pavilion, Suite 1.32, 5117 Centre Avenue, Pittsburgh, Pennsylvania 15213, USA. Phone: 412.624.009; Email: whitesidetl@upmc.edu.

1. Cerwenka A, Lanier LL. Natural killer cells, viruses and cancer. Nat Rev Immunol. 2001;1(1):41-49.

2. Herberman RB, Ortaldo JR. Natural killer cells: their roles in defenses against disease. Science.
1981;214(4516):24-30.

3. Kiessling R, Klein E, Pross H, Wigzell H. "Natural" killer cells in the mouse. II. Cytotoxic cells with specificity for mouse Moloney leukemia cells. Characteristics of the killer cell. Eur J Immunol. 1975;5(2):117-121.

4. Vivier E, Tomasello E, Baratin M, Walzer T, Ugolini S. Functions of natural killer cells. Nat Immunol. 2008;9(5):503-510.

5. Vitale M, et al. An historical overview: the discovery of how NK cells can kill enemies, recruit defense troops, and more. Front Immunol. 2019;10:1415.

6. Kärre K. Natural killer cell recognition of missing self. Nat Immunol. 2008;9(5):477-480.

7. Pahl J, Cerwenka A. Tricking the balance: NK cells in anti-cancer immunity. Immunobiology. 2017;222(1):11-20.

8. Hashemi E, Malarkannan S. Tissue-resident NK cells: development, maturation, and clinical relevance. Cancers (Basel). 2020;12(6):E1553.

9. Larsen SK, Gao Y, Basse PH. NK cells in the tumor microenvironment. Crit Rev Oncog. 2014;19(1-2):91-105.

10. Weinberg F, Ramnath N, Nagrath D. Reactive oxygen species in the tumor microenvironment: an overview. Cancers (Basel). 2019;11(8):E1191.

11. Yang $Y$, et al. Thioredoxin activity confers resistance against oxidative stress in tumorinfiltrating NK cells. J Clin Invest. 2020;130(10):5508-5522.

12. Mao Y, et al. IL-15 activates mTOR and primes stress-activated gene expression leading to prolonged antitumor capacity of NK cells. Blood. 2016;128(11):1475-1489.

13. Collet JF, Messens J. Structure, function, and mechanism of thioredoxin proteins. Antioxid Redox Signal. 2010;13(8):1205-1216. 